\title{
Adverse effects of extra-articular corticosteroid injections: a systematic review
}

\author{
Aaltien Brinks ${ }^{1 *}$, Bart W Koes ${ }^{1}$, Aloysius CW Volkers², Jan AN Verhaar ${ }^{3}$, Sita MA Bierma-Zeinstra ${ }^{1}$
}

\begin{abstract}
Background: To estimate the occurrence and type of adverse effects after application of an extra-articular (soft tissue) corticosteroid injection.

Methods: A systematic review of the literature was made based on a PubMed and Embase search covering the period 1956 to January 2010. Case reports were included, as were prospective and retrospective studies that reported adverse events of corticosteroid injection. All clinical trials which used extra-articular corticosteroid injections were examined. We divided the reported adverse events into major (defined as those needing intervention or not disappearing) and minor ones (transient, not requiring intervention).

Results: The search yielded 87 relevant studies:44 case reports, 37 prospective studies and 6 retrospective studies. The major adverse events included osteomyelitis and protothecosis; one fatal necrotizing fasciitis; cellulitis and ecchymosis; tendon ruptures; atrophy of the plantar fat was described after injecting a neuroma; and local skin effects appeared as atrophy, hypopigmentation or as skin defect. The minor adverse events effects ranged from skin rash to flushing and disturbed menstrual pattern. Increased pain or steroid flare after injection was reported in 19 studies. After extra-articular injection, the incidence of major adverse events ranged from $0-5.8 \%$ and that of minor adverse events from 0-81\%. It was not feasible to pool the risk for adverse effects due to heterogeneity of study populations and difference in interventions and variance in reporting.

Conclusion: In this literature review it was difficult to accurately quantify the incidence of adverse effects after extra-articular corticosteroid injection. The reported adverse events were relatively mild, although one fatal reaction was reported.
\end{abstract}

\section{Background}

In 1954 the first report on the effects of corticosteroids on healthy tissues appeared [1]. Local extra-articulair injections of glucocorticoid agents are currently used for rheumatic disorders including a wide spectrum of localised lesions of the tendons, enthesis, tendon sheaths, bursae, ligaments and fasciae as well as nerve compression syndromes [2]. Corticosteroid injections are frequently included as treatment option in clinical guidelines in the field of musculoskeletal disorders. Injectable corticosteroids are nowadays registered for local treatment of (rheumatic) arthritis, synovitis, bursitis, epicondylitis, tendonitis, neuromas, ganglion cysts, entrapment syndromes, fasciitis and back pain [3,4]. In 2006 Dutch pharmacists delivered

\footnotetext{
* Correspondence: a.brinks@erasmusmc.nl

'Department of General Practice Erasmus Medical Center P.O. Box 2040, 3000 CA The Netherlands

Full list of author information is available at the end of the article
}

208,380 prescriptions of injectable triamcinalone, representing $€ 2,867,000$ of the $€ 86,250,000$ total prescribed medication for the musculoskeletal system (3.3\%) [5] it is however not known how many of the injectable corticosteroids are given intra- or extra-articular. In a retrospective cohort study (on the five-year prognosis of trochanteric syndrome) $37 \%$ of the 164 cases were injected with corticosteroids [6]. A systematic therapeutic overview showed that $14-38 \%$ of patients with a tennis elbow in general practice were treated with corticosteroid injections [7]. In another study in general practice patients with tendosynovitis or nerve entrapment were injected with corticosteroid injection in $11 \%$ and $13 \%$ respectively [8]. Nevertheless there is only limited evidence to support the superiority of extra-articular glucocorticoid injections based on randomized trials [9]. Recently in RCT is reported efficacy of corticosteroid injections for trigger finger [10]. In addition, safety aspects of corticosteroid 
injections have so far not been adequately investigated by systematic reviews, except for complications associated with the use of corticosteroids in the treatment of athletic injuries [11]. Balanced decisions about healthcare interventions require evidence on harms as well as benefits [12]. Therefore, the aim of the present study was to estimate the occurrence of and describe the type of adverse effects due to extra-articular corticosteroid injections.

\section{Methods}

\section{Search}

The aim was to identify relevant articles describing adverse events of extra-articular corticosteroid injections. With our medical librarian (AV) we performed an extensive literature search in Pub Med and Embase covering the period 1956 until January 2010. The query was based on the definitions of terms related to adverse outcomes as stated in the Cochrane Handbook [13]. The key words and query comprised a combination of MESH terms and free-text words for injection locations (all joints, tendon, bursal, and ligamental location), with MESH terms for glucocorticosteroid products and the way of administration combined with all MESH terms and words related to adverse events. The search query is added as an additional file(see Additional file 1) In addition, the titles of references in the included articles or identified relevant reviews were checked for possibly relevant references.

Health professionals and patients can report suspicions of adverse drug reactions to the Netherlands Pharmacovigilance Centre 'Lareb'. The 'Lareb' collates adverse drug reaction data in the Netherlands; it performs this task on behalf of the Medicines Evaluation Board (MEB) [14]. The MEB is responsible for authorising and monitoring safe and effective medicinal products on the Dutch market, and shares in the responsibility for authorizing medicinal products throughout the European Union. Therefore we also contacted 'Lareb' for relevant data of adverse events reported after extra-articular corticosteroid injection.

\section{Inclusion criteria}

Two researchers read a share of the abstracts for inclusion in this review. Only studies that reported original patient material (e.g. case reports, case studies, cohort studies, clinical trials and case control studies) that reported on the occurrence of adverse events after intervention with local non-intra-articular corticosteroid injections were included. Studies concerning epidural injection and intramusculair injections were excluded. Because the adverse events of therapy are not always mentioned in the article abstracts, the full article of all relevant clinical trials were carefully read to find any reported adverse events [15].

\section{Data extraction and data syntheses}

Relevant study characteristics (including authors, year of publication, size of study population, type of intervention) were extracted. In addition, the type and number of adverse events were extracted, as was the follow-up time (prospective studies) and percentage lost to followup (as qualitative characteristics). Where possible, the percentage of persons with adverse events was calculated separately for major adverse events (defined by us as having a lasting effect, or needing intervention) and minor adverse events (defined as transient ones not needing intervention). In the clinical trials, the frequency percentage of adverse events was calculated only for the group receiving intervention with corticosteroids. Further, a summary of the frequency of such adverse events was based on prospective studies only. Only in the case of homogenous definitions of adverse effects, interventions and study populations we did consider pooling the risk for adverse-effects. The types of major and minor adverse events were summarized separately.

The data received from 'Lareb' were also analysed separately. These data include the indication for the corticosteroid injections, gender of the patient, and route of administration. We only report here on adverse events of injections that we know for certain were applied extra-articularly.

In this review we used the terminology for adverse drug reactions noted in the Cochrane Handbook [13]. We used the term 'adverse event' for an unfavourable outcome that occurs during or after the use of a drug or other intervention but is not necessarily caused by it. 'Adverse effect' is used for an adverse event for which the causal relation between the intervention and the event is at least a reasonable possibility. Adverse drug reaction' was used for an adverse effect specific to a drug. 'Side effect' was defined by any unintended effect, adverse or beneficial, of a drug that occurs at doses normally used for treatment, and 'complications' as an adverse events or effects following surgical and other invasive interventions. In the data-extraction, however, we report the terminology used by the authors.

\section{Results \\ Output}

The search (1956 to January 2010) yielded 1,313 articles. After reading the abstracts, there were 290 possibly relevant articles on adverse effects after extra-articular injection. After studying the full-text articles and references of the included articles and relevant reviews, there were 87 relevant articles, i.e. 44 case reports, 37 prospective studies and 6 retrospective studies reporting on the adverse events of extra-articular local corticosteroid injections. 
'Lareb' provided a list of reported adverse events after triamcinolone injections.

\section{Types of adverse events Case studies}

Only two articles mentioned an adverse event after a corticosteroid injection in the bursa round the hip. One of these studies reported a complication after a ten-fold higher dose was accidentally given [16], and the other reported a case of a necrotizing fasciitis after a corticosteroid injection in the trochanteric bursa - which proved to be a lethal complication [17]. Necrotizing fasciitis after corticosteroid injection for trigger finger was presented as another severe complication [18]. Six studies described hypopigmentation of the skin [19-24], and two studies described atrophy of the skin [21,23]. Atrophy of the plantar fat pad was reported after injecting an interdigital neuroma, and another group reported perilymphatic atrophy $[25,26]$. Atrophy of the skin and subcutaneous fat with hyperpigmentation was described in one patient after intralesional injection of a neuroma at the feet [27]. A skin defect was observed after two injections of triamcinolone injected into a hypertrophic scar [28]. Osteomyelitis of the humerus was reported after three injections with hydrocortisone for a tennis elbow [29]. Osteomyelitis of the calcaneus was reported after an injection for plantar fasciitis [30]. Localized abscess containing Staphylococcus aureus was described after injection of corticosteroid for the treatment of chronic tendinitis of the Achilles tendon [31]. Another article mentioned a sterile abscess after injecting a patient with a plantar fasciitis [32]. Protothecosis (a rare infection caused by an achlorophyllic algae) was seen in two patients after intralesional injections with corticosteroids [33]. Atypical mycobacterium soft tissue infection was reported after corticosteroid injection for de Quervain's disease [34]. An allergic reaction was reported after giving an injection to a patient who had tendonitis [35]. A tendon rupture of the hand was described after an injection into the carpal tunnel, and a tendon rupture after an injection for tennis elbow $[36,37]$. A delayed flexor superficiales and profundus rupture occurred after a steroid injection for trigger finger [38]. Seven weight lifters presented at the hospital with ruptured patellar tendon, they all had a history of multiple local steroid injections [39]. A rupture of the Achilles tendon associated with corticosteroid injections was reported in three studies [40-42]. Another case report described an avulsion of the calcaneal tendon after steroid injections administered because of an acute flare-up of rheumatoid arthritis [43]. One study described thirteen patients who developed 15 ruptured tendons subsequent to injection of a depository steroid in or around the tendons injected [44]. One study reported ischemia of the hand after carpal tunnel injection and one study after a corticosteroid injection for de Quervain tenosynovitis $[45,46]$. Nerve injury after steroid injection for carpal tunnel syndrome is described in 3 studies [47-49]. Soft tissue calcifications were reported as a complication due to adjusted materials in the solvent or due to an accumulation of insoluble steroid [50-52].

\section{Prospective studies}

Of the 37 prospective studies, 11 reported no adverse effects. Hypopigmentation was reported in three studies [53-55]. Atrophy was described in four studies [54,56-58]. Increased or persistent pain after injection or pain at the site of injection was described in 19 studies. Adverse events not mentioned in the case reports were flushes and disturbance in menstrual pattern [59]. Cellulitis, ecchymosis and subcutaneous nodule were three other symptoms not mentioned earlier in the case reports [55,60]. Table 1 presents information on the minor and major adverse events in the prospective studies.

\section{Retrospective studies}

In one retrospective study septic bursitis was described after corticosteroid injection in traumatic olecranon bursitis [61]. Tachon's syndrome (subacute back pain and/ or thoracic pain following local injections of corticosteroids) was reported in one study [62]. Table 2 presents information on the adverse events in the retrospective studies.

\section{Lareb Institute}

The following adverse events were registered by the 'Lareb' institute following extra-articular indications: after corticosteroid injection for bursitis trochanterica flushing was reported, after injection for tennis elbow, rash, menstrual disorder, and skin depigmentation and in one patient dyspnoea and eyelid ptosis were reported. In one patient hallucination, increased intracranial and intraocular pressure, and paresis occurred after corticosteroid injection for a calcaneal spur. In another patient, after corticosteroid injection for carpal tunnel syndrome hirsutism, nail changes and vaginal hemorrhage were reported. After injection for trigger finger an allergic skin reaction was observed. Reported adverse events after corticosteroid injections for tendonitis were: anaphylactic reaction in one patient, erythema and skin atrophy in another, and rash and tendon disorder in the third patient.

Frequency of adverse events Due to the heterogeneity of the study populations, the type of interventions, the uncertain causality of the reported reaction with the administered corticosteroid injection and the impossibility to count risk differences in all the studies, we refrained from pooling the risk for adverse-effects. Minor adverse events were: 
Table 1 Summary of the included prospective studies

\begin{tabular}{|c|c|c|c|c|c|c|}
\hline $\begin{array}{l}\text { Author, Year of } \\
\text { publication }\end{array}$ & $\begin{array}{l}\text { Type of } \\
\text { study, } \\
\text { number } \\
\text { of cases }\end{array}$ & Indication & $\begin{array}{l}\text { Corticosteroid injection } \\
\text { agent (no. of injected } \\
\text { cases, sides) }\end{array}$ & $\begin{array}{l}\text { Methods of reporting } \\
\text { adverse events }\end{array}$ & $\begin{array}{l}\text { Complications reported } \\
\text { Major/minor Period of } \\
\text { time }\end{array}$ & $\begin{array}{l}\text { Follow-up } \\
\text { (\%) }\end{array}$ \\
\hline Rompe 2009 [69] & $\begin{array}{l}\text { RCT } \\
(229)\end{array}$ & GTPS & $\begin{array}{l}\text { Prednisolon } 25 \mathrm{mg} / \\
\text { meaverin } 0.5 \%(75)\end{array}$ & $\begin{array}{l}\text { In method section: adverse } \\
\text { effects were recorded by the } \\
\text { physician Results: } \\
\text { summarised in a table }\end{array}$ & $\begin{array}{l}\text { Minor: increased or } \\
\text { radiating pain: } 44 \% \text {, skin } \\
\text { irritation 3\%, swelling } 9 \%\end{array}$ & $\begin{array}{l}92 \% \text { after } \\
15 \text { months }\end{array}$ \\
\hline Gunter 2004 [70] & $\mathrm{RCT}(18)$ & $\begin{array}{l}\text { lliotibial } \\
\text { band friction } \\
\text { syndrome }\end{array}$ & $\begin{array}{l}\text { Methylprednisolone acetate } \\
40 \mathrm{mg} / \text { /ignocaine } 1 \% \text { (9) }\end{array}$ & $\begin{array}{l}\text { In method section: side } \\
\text { effects/adverse reactions: are } \\
\text { reported in both } \\
\text { intervention groups as a } \\
\text { separate issue Results: } \\
\text { mentioned as a separate } \\
\text { issue }\end{array}$ & $\begin{array}{l}\text { No side-effects after } 7 \text { and } \\
14 \text { days }\end{array}$ & $\begin{array}{l}100 \% \text { at } \\
2 \text { weeks }\end{array}$ \\
\hline Chao 2009 [71] & $\mathrm{RCT}(97)$ & $\begin{array}{l}\text { Trigger } \\
\text { thumb }\end{array}$ & Triamcenolon 10 mg (42) & $\begin{array}{l}\text { In method section: no } \\
\text { information Results: } \\
\text { mentioned in a sentence }\end{array}$ & $\begin{array}{l}\text { Minor: } 2.2 \% \text { had pain after } \\
1 \text { month }\end{array}$ & $\begin{array}{l}100 \% \text { after } \\
12 \text { months }\end{array}$ \\
\hline Peters 2008 [72] & $\mathrm{RCT}(50)$ & $\begin{array}{l}\text { Trigger } \\
\text { finger }\end{array}$ & $\begin{array}{l}\text { Triamcinolone acetonide: } 10, \\
1 \text { or } 2 \text { injections (41) }\end{array}$ & $\begin{array}{l}\text { In method section: adverse } \\
\text { event as secondary outcome } \\
\text { Results: mentioned as a } \\
\text { separate issue }\end{array}$ & $\begin{array}{l}\text { Minor: hot flushes } 22 \% \text {, } \\
\text { steroid-flare } 14.6 \%\end{array}$ & $\begin{array}{l}82 \% \text { after } \\
12 \text { months } \\
\text { follow-up }\end{array}$ \\
\hline $\begin{array}{l}\text { Jianmongkol } 2007 \\
\text { [73] }\end{array}$ & $\begin{array}{l}\text { RCT } \\
(101)\end{array}$ & $\begin{array}{l}\text { Trigger } \\
\text { finger, } 2 \\
\text { types of } \\
\text { injection } \\
\text { therapy } \\
\text { were } \\
\text { compared } \\
(48 / 53)\end{array}$ & $\begin{array}{l}\text { Triamcinolone } 10 \mathrm{mg} / \\
\text { lidocaine (101) }\end{array}$ & $\begin{array}{l}\text { In method section: no } \\
\text { information Results: reported } \\
\text { in one sentence }\end{array}$ & No complications & $\begin{array}{l}\text { Follow-up } 6 \\
\text { weeks (\% } \\
\text { lost to } \\
\text { follow-up } \\
\text { not } \\
\text { mentioned) }\end{array}$ \\
\hline Goldfarb 2007 [74] & $\begin{array}{l}\text { RCT } \\
(154)\end{array}$ & $\begin{array}{l}\text { Trigger } \\
\text { finger or De } \\
\text { Quervain's } \\
\text { tenosynovitis }\end{array}$ & $\begin{array}{l}\text { Methylprednisolone acetate } \\
40 \mathrm{mg} / \text { /idocaine } 1 \% / \\
\text { bupivacaine } 0.5 \% \text { (154) }\end{array}$ & $\begin{array}{l}\text { In method section: } \\
\text { incidence of post injection } \\
\text { pain flare was the aim of } \\
\text { the study, no other } \\
\text { complications are monitored } \\
\text { Results: flare reaction } \\
\text { mentioned as a separate } \\
\text { issue }\end{array}$ & $\begin{array}{l}\text { Minor: in } 33 \% \text { increase in } \\
\text { pain score of } 2 \text { points or } \\
\text { more (VAS scale 0-10). }\end{array}$ & $\begin{array}{l}81 \% \text { follow- } \\
\text { up after } 1 \\
\text { and } 6 \\
\text { weeks }\end{array}$ \\
\hline $\begin{array}{l}\text { Baumgarten } 2007 \\
{[75]}\end{array}$ & $\mathrm{RCT}(59)$ & $\begin{array}{l}\text { Trigger } \\
\text { finger in } \\
\text { diabetics } \\
\text { versus non } \\
\text { diabetics }\end{array}$ & $\begin{array}{l}\text { Betamethasone } 6 \mathrm{mg} / \\
\text { lidocaine } 1 \%(44)\end{array}$ & $\begin{array}{l}\text { In method section: in follow- } \\
\text { up section: complications } \\
\text { related to treatment were } \\
\text { reported Results: } \\
\text { complications reported as a } \\
\text { separate issue }\end{array}$ & $\begin{array}{l}\text { No adverse events at } 6 \\
\text { weeks, } 3 \text { months and } 1 \\
\text { year }\end{array}$ & $\begin{array}{l}98 \% \text { follow- } \\
\text { up at } 12 \\
\text { month } \\
\text { (range 13- } \\
41 \text { months) }\end{array}$ \\
\hline Kazuki 2006 [76] & $\begin{array}{l}\text { Pros } \\
(100)\end{array}$ & $\begin{array}{l}\text { Trigger } \\
\text { finger }\end{array}$ & $\begin{array}{l}\text { Betamethasone } 2.5 \mathrm{mg} / \\
\text { lidocaine } 1 \% \text { (129 fingers) }\end{array}$ & $\begin{array}{l}\text { In method section: not } \\
\text { mentioned. Results: one } \\
\text { sentence: no complications } \\
\text { of steroid injections were } \\
\text { observed }\end{array}$ & $\begin{array}{l}\text { No complications after } 6 \\
\text { months }\end{array}$ & $\begin{array}{l}100 \% \\
\text { follow-up } \\
\text { after } \\
6 \text { months } \\
\text { (range 1-42) }\end{array}$ \\
\hline Gurcay 2009 [77] & $\mathrm{RCT}(36)$ & $\begin{array}{l}\text { Carpal } \\
\text { tunnel } \\
\text { syndrome }\end{array}$ & Betamethasone 6 mg (18) & $\begin{array}{l}\text { In method section: not } \\
\text { mentioned Results: no } \\
\text { complications or side effects } \\
\text { to treatment were observed }\end{array}$ & No side effects & $\begin{array}{l}100 \% \\
\text { follow-up } \\
\text { after } \\
3 \text { months }\end{array}$ \\
\hline $\begin{array}{l}\text { Nalamachu } 2006 \\
\text { [78] }\end{array}$ & $\mathrm{RCT}(40)$ & $\begin{array}{l}\text { Carpal } \\
\text { tunnel } \\
\text { syndrome }\end{array}$ & $\begin{array}{l}\text { Methylprednisolone } 40 \text { mg/ } \\
\text { lidocaine } 1 \%(20)\end{array}$ & $\begin{array}{l}\text { In method section: adverse } \\
\text { events were classified } \\
\text { according to MedDRA and } \\
\text { the incidence of treatment } \\
\text { emergent events was } \\
\text { summarized Results: adverse } \\
\text { events were described }\end{array}$ & $\begin{array}{l}\text { Minor: numbness (5\%), } \\
\text { local pain (5\%), tingling in } \\
\text { hands at } 4 \text { weeks (5\%) }\end{array}$ & $\begin{array}{l}85 \% \text { follow- } \\
\text { up after } \\
4 \text { weeks }\end{array}$ \\
\hline $\begin{array}{l}\text { Dammers } 2005 \\
\text { [79] }\end{array}$ & $\begin{array}{l}\text { RCT } \\
(132)\end{array}$ & $\begin{array}{l}\text { Carpal } \\
\text { tunnel } \\
\text { syndrome }\end{array}$ & $\begin{array}{l}\text { Methylprednisolone } 20 \text { mg } \\
\text { (45), } 40 \text { mg (43), } 60 \text { mg (44) } \\
\text { with lidocaine } 10 \text { mg }\end{array}$ & $\begin{array}{l}\text { In method section: not } \\
\text { mentioned Results: no side } \\
\text { effects were recorded }\end{array}$ & $\begin{array}{l}\text { No side-effects after } 1 \text { and } \\
12 \text { months }\end{array}$ & $\begin{array}{l}97 \% \text { follow- } \\
\text { up after } \\
12 \text { months }\end{array}$ \\
\hline
\end{tabular}


Table 1 Summary of the included prospective studies (Continued)

\begin{tabular}{|c|c|c|c|c|c|c|}
\hline Hui 2005 [60] & $\mathrm{RCT}(50)$ & $\begin{array}{l}\text { Carpal } \\
\text { tunnel } \\
\text { syndrome }\end{array}$ & $\begin{array}{l}\text { Methylprednisolon } 15 \mathrm{mg} \\
(25)\end{array}$ & $\begin{array}{l}\text { In method section: surgical } \\
\text { complications are assessed } \\
\text { after one week, no other } \\
\text { adverse events mentioned. } \\
\text { Results: one patient with } \\
\text { cellulitis is reported and four } \\
\text { patients with pain at the } \\
\text { injection side }\end{array}$ & $\begin{array}{l}\text { Minor: pain at injection } \\
\text { side } 16 \% \text { Major: cellulitis } \\
4 \%\end{array}$ & $\begin{array}{l}100 \% \text { at } \\
6 \text { and } \\
20 \text { weeks }\end{array}$ \\
\hline Wong 2005 [80] & $\mathrm{RCT}(40)$ & $\begin{array}{l}\text { Carpal } \\
\text { tunnel } \\
\text { syndrome }\end{array}$ & $\begin{array}{l}\text { Methylprednisolone } 15 \mathrm{mg} \\
\text { single dose (20) or double } \\
\text { dose (20) }\end{array}$ & $\begin{array}{l}\text { In method section: any side } \\
\text { effects were recorded at } 8 \text {, } \\
24 \text { and } 40 \text { weeks Results: } \\
\text { reported as a sentence at } \\
\text { the end of the result section }\end{array}$ & $\begin{array}{l}\text { Minor: local pain (30\% in } \\
20 \text { mg group and } 10 \% \text { in } \\
20 \text { mg group) }\end{array}$ & $\begin{array}{l}100 \% \\
\text { follow-up at } \\
8 \text { weeks }\end{array}$ \\
\hline Agarwal 2005 [81] & Pros (48) & $\begin{array}{l}\text { Carpal } \\
\text { tunnel } \\
\text { syndrome }\end{array}$ & $\begin{array}{l}\text { Methylprednisolone acetate } \\
40 \text { mg/xylocaine } 2 \% \\
\text { (67 hands) }\end{array}$ & $\begin{array}{l}\text { In method section: not } \\
\text { mentioned Results: at the } \\
\text { end of the result section } \\
\text { adverse effects were } \\
\text { mentioned }\end{array}$ & $\begin{array}{l}\text { Minor: mild discoloration of } \\
\text { the skin over the injection } \\
\text { site }(6 \%)\end{array}$ & $\begin{array}{l}100 \% \\
\text { follow-up } \\
\text { after } 3 \\
\text { months, } \\
78 \% \text { after } \\
12 \text { months }\end{array}$ \\
\hline Ly-Pen 2005 [82] & $\begin{array}{l}\mathrm{RCT} \\
(163)\end{array}$ & $\begin{array}{l}\text { Carpal } \\
\text { tunnel } \\
\text { syndrome }\end{array}$ & $\begin{array}{l}\text { Paramethasone acetonide } \\
20 \text { mg ( } 82,69 \text { wrists } \\
\text { required second injection) }\end{array}$ & $\begin{array}{l}\text { In method section: not } \\
\text { mentioned Results: safety } \\
\text { and tolerability was a } \\
\text { separate chapter }\end{array}$ & No relevant side-effects & $\begin{array}{l}79.5 \% \\
\text { follow-up at } \\
12 \text { months }\end{array}$ \\
\hline Sevim 2004 [83] & $\begin{array}{l}\mathrm{RCT} \\
(120)\end{array}$ & $\begin{array}{l}\text { Carpal } \\
\text { tunnel } \\
\text { syndrome }\end{array}$ & Betamethasone 6 mg. (60) & $\begin{array}{l}\text { In method section: not } \\
\text { mentioned Results: } \\
\text { complications and side } \\
\text { effects are described }\end{array}$ & $\begin{array}{l}\text { Minor: moderate pain } \\
\text { lasting less than } 24 \text { hours } \\
\text { after injection }(3.4 \%) \text {, } \\
\text { haematoma }(1.7 \%)\end{array}$ & $\begin{array}{l}90 \% \text { follow- } \\
\text { up at } \\
11 \text { months } \\
\text { follow-up } \\
\text { (range } 9 \text { to } \\
14 \text { months) }\end{array}$ \\
\hline $\begin{array}{l}\text { Armstrong } 2004 \\
{[84]}\end{array}$ & $\mathrm{RCT}(81)$ & $\begin{array}{l}\text { Carpal } \\
\text { tunnel } \\
\text { syndrome }\end{array}$ & $\begin{array}{l}\text { Betamethasone } 6 \mathrm{mg} / \\
\text { lidocaine } 1 \% \text { ( } 43 \text { with a } \\
\text { total of } 364 \text { injections) }\end{array}$ & $\begin{array}{l}\text { In method section: side } \\
\text { effects and complications } \\
\text { are recorded Results: adverse } \\
\text { effects described }\end{array}$ & $\begin{array}{l}\text { Minor: severe pain after } \\
\text { injection (5\%), acute } \\
\text { transient sympathetic } \\
\text { reaction after injection (2\%) }\end{array}$ & $\begin{array}{l}89 \% \text { follow- } \\
\text { up after } 18 \\
\text { months }\end{array}$ \\
\hline Wong 2001 [85] & $\mathrm{RCT}(62)$ & $\begin{array}{l}\text { Carpal } \\
\text { tunnel } \\
\text { syndrome }\end{array}$ & $\begin{array}{l}\text { Methylprednisolone } 15 \mathrm{mg} \\
\text { (30) }\end{array}$ & $\begin{array}{l}\text { In method section: any side } \\
\text { effects were recorded by } \\
\text { telephone interview Results: } \\
\text { summarized in a table }\end{array}$ & Minor: injection pain (6.7\%) & $\begin{array}{l}100 \% \text { after } \\
12 \text { weeks }\end{array}$ \\
\hline Kalaci 2009 [64] & $\begin{array}{l}\mathrm{RCT} \\
(100)\end{array}$ & $\begin{array}{l}\text { Plantar } \\
\text { fasciitis }\end{array}$ & Triamcinolone 20 mg (50) & $\begin{array}{l}\text { In method section: not } \\
\text { mentioned Results: } \\
\text { description of the side } \\
\text { effects not found }\end{array}$ & $\begin{array}{l}\text { No side effects or } \\
\text { complications All of the } \\
\text { patients found the } \\
\text { injection painful }\end{array}$ & $\begin{array}{l}100 \% \text { after } \\
6 \text { months }\end{array}$ \\
\hline Porter 2005 [86] & $\begin{array}{l}\mathrm{RCT} \\
(132)\end{array}$ & $\begin{array}{l}\text { Plantar } \\
\text { fasciopathy }\end{array}$ & $\begin{array}{l}\text { Betamethason } 5.7 \text { mg/ } \\
\text { lignocaine 1\% (64) }\end{array}$ & $\begin{array}{l}\text { In method section: patients } \\
\text { were asked to report any } \\
\text { possible side effects at } 3 \\
\text { and } 12 \text { months Results: no } \\
\text { infections or rupture are } \\
\text { found, description of the } \\
\text { side effects }\end{array}$ & $\begin{array}{l}\text { Minor: post-injection pain } \\
(12.5 \%) \text { that required } \\
\text { analgesia and/or ice } \\
\text { application }\end{array}$ & $\begin{array}{l}95 \% \text { follow- } \\
\text { up at } 12 \\
\text { months }\end{array}$ \\
\hline Genc 2005 [87] & Pros (30) & $\begin{array}{l}\text { Plantar } \\
\text { fasciitis }\end{array}$ & $\begin{array}{l}\text { Methylprednisolone } 20 \text { mg/ } \\
\text { prolocaine 2\% (47 heels) }\end{array}$ & $\begin{array}{l}\text { In method section: } \\
\text { ultrasonografy measurement } \\
\text { of the facia at } 1 \text { and } 6 \\
\text { months Results: reported as } \\
\text { one sentence }\end{array}$ & No rupture observed & $\begin{array}{l}100 \% \\
\text { follow-up at } \\
6 \text { months }\end{array}$ \\
\hline $\begin{array}{l}\text { Lindenhovius } 2008 \\
\text { [88] }\end{array}$ & $\mathrm{RCT}(64)$ & $\begin{array}{l}\text { Lateral } \\
\text { elbow pain }\end{array}$ & $\begin{array}{l}\text { Dexamethasone } 4 \mathrm{mg} / \\
\text { lidocaine } 1 \%(31)\end{array}$ & $\begin{array}{l}\text { In method section: not } \\
\text { mentioned Results: adverse } \\
\text { events are described }\end{array}$ & $\begin{array}{l}\text { Minor: discoloration of skin } \\
3.2 \% \text { increased elbow pain } \\
3.2 \%\end{array}$ & $\begin{array}{l}77 \% \text { after } \\
1 \text { and } 6 \\
\text { months }\end{array}$ \\
\hline Tonks 2007 [53] & $\mathrm{RCT}(48)$ & $\begin{array}{l}\text { Epicondylitis } \\
\text { lateralis }\end{array}$ & $\begin{array}{l}\text { Triamcinolone acetonide } \\
10 \text { mg/lignocaine } 2 \%(24)\end{array}$ & $\begin{array}{l}\text { In method section: } \\
\text { complications of treatment } \\
\text { were one of the outcome } \\
\text { measurements Result } \\
\text { section: complications are } \\
\text { described }\end{array}$ & $\begin{array}{l}\text { Major: skin depigmentation } \\
\text { and atrophy in } 4 \% \text { after } 7 \\
\text { weeks }\end{array}$ & $\begin{array}{l}77 \% \text { follow- } \\
\text { up at } \\
7 \text { weeks }\end{array}$ \\
\hline
\end{tabular}


Table 1 Summary of the included prospective studies (Continued)

\begin{tabular}{|c|c|c|c|c|c|c|}
\hline Bisset 2006 [54] & $\begin{array}{l}\mathrm{RCT} \\
(198)\end{array}$ & $\begin{array}{l}\text { Tennis } \\
\text { elbow }\end{array}$ & $\begin{array}{l}\text { Triamcinolone } 10 \mathrm{mg} / \\
\text { lidocaine } 1 \%(65)\end{array}$ & $\begin{array}{l}\text { In method section: not } \\
\text { mentioned Results: side } \\
\text { effects were mentioned in a } \\
\text { separate section }\end{array}$ & $\begin{array}{l}\text { Minor: pain (18.5\%). Major: } \\
\text { loss of skin pigment (3\%), } \\
\text { atrophy of subcutaneous } \\
\text { tissue }(1.5 \%)\end{array}$ & $\begin{array}{l}100 \% \\
\text { follow-up in } \\
\text { injection } \\
\text { group at } \\
12 \text { months }\end{array}$ \\
\hline Wang 2003 [89] & Pros (94) & $\begin{array}{l}\text { Hand and } \\
\text { elbow } \\
\text { injections }\end{array}$ & $\begin{array}{l}\text { Betamethasone/lidocaine } \\
1 \%\end{array}$ & $\begin{array}{l}\text { In method section: } \\
\text { registration of pain levels } \\
\text { after injection of } \\
\text { corticosteroid to hand and } \\
\text { elbow was the aim of the } \\
\text { study, no other side effects } \\
\text { were recorded Results: post } \\
\text { injection pain is shown in } \\
\text { table and list }\end{array}$ & $\begin{array}{l}\text { Minor: } 50 \% \text { increased post- } \\
\text { injection pain during } 1.2 \\
\text { days }\end{array}$ & $\begin{array}{l}71 \% \text { follow- } \\
\text { up at } 5 \\
\text { days }\end{array}$ \\
\hline Smidt 2002 [90] & $\begin{array}{l}\mathrm{RCT} \\
(185)\end{array}$ & $\begin{array}{l}\text { Epicondylitis } \\
\text { lateralis }\end{array}$ & $\begin{array}{l}\text { Triamcinolone acetonide } 10 \\
\text { mg/lidocaine (62) }\end{array}$ & $\begin{array}{l}\text { In method section: details of } \\
\text { any adverse effects were } \\
\text { reported on standardised } \\
\text { forms Results: adverse } \\
\text { effects summarized in a } \\
\text { table }\end{array}$ & $\begin{array}{l}\text { Minor: facial flush (3\%), skin } \\
\text { irritation (5\%), red swollen } \\
\text { elbow (3\%), change of skin } \\
\text { colour (11\%), other not } \\
\text { specified side-effects (13\%) }\end{array}$ & $\begin{array}{l}96 \% \text { follow- } \\
\text { up at } 52 \\
\text { weeks }\end{array}$ \\
\hline Jensen 2001 [91] & $\mathrm{RCT}(30)$ & $\begin{array}{l}\text { Tennis } \\
\text { elbow }\end{array}$ & $\begin{array}{l}\text { Methylprednisolone } 20 \text { mg/ } \\
\text { lidocaine 1\% (16) }\end{array}$ & $\begin{array}{l}\text { In method section: daily } \\
\text { pain registration for six } \\
\text { weeks Results: described in } \\
\text { result section }\end{array}$ & $\begin{array}{l}\text { Minor: pain increase after } \\
\text { injection }(81 \%)\end{array}$ & $\begin{array}{l}100 \% \\
\text { follow-up } \\
6 \text { weeks }\end{array}$ \\
\hline Hay 1999 [57] & $\begin{array}{l}\mathrm{RCT} \\
(164)\end{array}$ & $\begin{array}{l}\text { Tennis } \\
\text { elbow }\end{array}$ & $\begin{array}{l}\text { Methylprednisolone } 20 \text { mg/ } \\
\text { lignocaine (51) }\end{array}$ & $\begin{array}{l}\text { In method section: } \\
\text { complications of treatment } \\
\text { is one of the secondary } \\
\text { outcome Results: described } \\
\text { in a separate section side } \\
\text { effects }\end{array}$ & $\begin{array}{l}\text { Major: local skin atrophy in } \\
\text { the overall group ( } 3 \text { of } \\
111) \text {, one with steroids } \\
(1.9 \%)\end{array}$ & $\begin{array}{l}100 \% \\
\text { follow-up at } \\
12 \text { months }\end{array}$ \\
\hline Stahl 1997 [92] & $\mathrm{RCT}(58)$ & $\begin{array}{l}\text { Medial } \\
\text { epicondylitis }\end{array}$ & $\begin{array}{l}\text { Methylprednisolone } 40 \text { mg/ } \\
\text { lidocaine (30) }\end{array}$ & $\begin{array}{l}\text { In method section: } \\
\text { interviews and physical } \\
\text { examination for possible } \\
\text { local complications Results: } \\
\text { complications are reported } \\
\text { in a separate part }\end{array}$ & $\begin{array}{l}\text { Major: non reported Minor: } \\
\text { facial flushing in one } \\
\text { female patient }\end{array}$ & $\begin{array}{l}100 \% \\
\text { follow-up } \\
12 \text { months }\end{array}$ \\
\hline Verhaar 1995 [93] & $\begin{array}{l}\mathrm{RCT} \\
(106)\end{array}$ & $\begin{array}{l}\text { Tennis } \\
\text { elbow }\end{array}$ & Triamcinolone 1\% (53) & $\begin{array}{l}\text { In method section: side } \\
\text { effects not specified Results: } \\
\text { no infection or skin } \\
\text { hypopigmentation }\end{array}$ & $\begin{array}{l}\text { No side effects in the } \\
\text { injection group }\end{array}$ & $\begin{array}{l}100 \% \text { follow } \\
\text { up after } 12 \\
\text { months }\end{array}$ \\
\hline Price 1991 [58] & $\begin{array}{l}\mathrm{RCT} \\
(145)\end{array}$ & $\begin{array}{l}\text { Tennis } \\
\text { elbow }\end{array}$ & $\begin{array}{l}\text { Triamcinolone } 10 \mathrm{mg} / \\
\text { lignocaine } 1 \% \text { or } \\
\text { Hydrocortisone } 25 \mathrm{mg} / \\
\text { lignocaine } 1 \% \text { compared } \\
\text { with lignocaine } 1 \% \text { or with } \\
\text { Triamcinolone } 20 \mathrm{mg} \text {. } \\
\text { Second study Triamcinolone } \\
10 \text { mg versus } 20 \text { mg }\end{array}$ & $\begin{array}{l}\text { In method section: severe } \\
\text { post-injection pain and skin } \\
\text { atrophy were noted Results: } \\
\text { table with the adverse } \\
\text { effects }\end{array}$ & $\begin{array}{l}\text { Minor: post-injection pain } \\
(11 \%-58 \%) . \text { Major: skin } \\
\text { atrophy }(17 \%-40 \%)\end{array}$ & $\begin{array}{l}\text { Follow-up } \\
\text { at } 24 \text { weeks } \\
\text { (\% lost to } \\
\text { follow-up } \\
\text { not clear) }\end{array}$ \\
\hline $\begin{array}{l}\text { Jirarattanaphochai } \\
2004 \text { [55] }\end{array}$ & $\begin{array}{l}\mathrm{RCT} \\
(160)\end{array}$ & $\begin{array}{l}\text { De } \\
\text { Quervain's } \\
\text { tenosynovitis }\end{array}$ & $\begin{array}{l}\text { Triamcinolone acetonide } \\
10 \text { mg (100) }\end{array}$ & $\begin{array}{l}\text { In method section: the } \\
\text { adverse events reported at } 3 \\
\text { weeks, } 6 \text { and } 12 \text { months are } \\
\text { secondary outcome } \\
\text { measurements Results: } \\
\text { adverse effects are } \\
\text { mentioned in a table }\end{array}$ & $\begin{array}{l}\text { Minor: post-injection pain } \\
(13 \%), \text { subcutaneous } \\
\text { nodule ( } 2.5 \%) \text {, ecchymosis } \\
\text { (1.3\%). Major: skin } \\
\text { hypopigmentation }(1.3 \%)\end{array}$ & $\begin{array}{l}100 \% \\
\text { follow-up, } \\
3 \% \text { lost } \\
\text { between } \\
6 \text { and } \\
12 \text { months }\end{array}$ \\
\hline Avci 2002 [94] & CТ (19) & $\begin{array}{l}\text { Pregnant or } \\
\text { lactating } \\
\text { women with } \\
\text { De } \\
\text { Quervain's } \\
\text { tenosynovitis }\end{array}$ & $\begin{array}{l}\text { Methylprednisolone } 10 \mathrm{mg} \\
\text { (10) }\end{array}$ & $\begin{array}{l}\text { In method section: not } \\
\text { mentioned Result section: } \\
\text { not specified }\end{array}$ & $\begin{array}{l}\text { No side effects or local } \\
\text { complications of } \\
\text { corticosteroid injection } \\
\text { were noted }\end{array}$ & $\begin{array}{l}100 \% \text { follow } \\
\text { (range } 9- \\
17 \text { months) }\end{array}$ \\
\hline
\end{tabular}


Table 1 Summary of the included prospective studies (Continued)

\begin{tabular}{|c|c|c|c|c|c|c|}
\hline Anderson 1991[95] & Pros (56) & $\begin{array}{l}\text { De Quervain } \\
\text { tenosynovitis }\end{array}$ & $\begin{array}{l}\text { Methylprednisolone acetate } \\
40 \text { mg }\end{array}$ & $\begin{array}{l}\text { In method section: adverse } \\
\text { reaction were recorded, } \\
\text { particularly signs of atrophy } \\
\text { Result: adverse reactions are } \\
\text { summarised in a table }\end{array}$ & $\begin{array}{l}\text { Minor; pain 18\%, pain, } \\
\text { swelling, heat } 5 \% \\
\text { ecchymosis } 9 \% \text { temporary } \\
\text { radial nerve paresthesia 2\% } \\
\text { vasovagal reaction } 2 \% \\
\text { Major: subcutaneous fat } \\
\text { atrophy } 16 \%\end{array}$ & $\begin{array}{l}95 \% \text { follow- } \\
\text { up at } \\
4.2 \text { years }\end{array}$ \\
\hline $\begin{array}{l}\text { Crawford } 1999 \\
\text { [96] }\end{array}$ & $\begin{array}{l}\mathrm{RCT} \\
(106)\end{array}$ & Heel pain & $\begin{array}{l}\text { Methylprednisolone } 25 \text { mg } \\
\text { (53) }\end{array}$ & $\begin{array}{l}\text { In methods and results } \\
\text { sections: not mentioned }\end{array}$ & No side-effects reported & $\begin{array}{l}52 \% \text { follow- } \\
\text { up after } \\
6 \text { months }\end{array}$ \\
\hline Capasso 1997 [97] & $\begin{array}{l}\mathrm{RCT} \\
(116)\end{array}$ & $\begin{array}{l}\text { Patellar } \\
\text { tendopathy }\end{array}$ & $\begin{array}{l}\text { Methylprednisolone } 40 \text { mg/ } \\
\text { lignocaine (39) }\end{array}$ & $\begin{array}{l}\text { In method section: not } \\
\text { mentioned Results: } \\
\text { acceptability of treatment is } \\
\text { separately discussed in a } \\
\text { chapter }\end{array}$ & $\begin{array}{l}\text { Minor: burning sensation } \\
(10.3 \%) \text { injection pain } \\
(5.1 \%)\end{array}$ & $\begin{array}{l}82 \% \text { follow- } \\
\text { up after } \\
12 \text { months }\end{array}$ \\
\hline Mens 1998 [59] & $\begin{array}{l}\text { Pros } \\
(77 \text { Q })\end{array}$ & $\begin{array}{l}\text { Musculo- } \\
\text { skeletal } \\
\text { disease }\end{array}$ & $\begin{array}{l}\text { Triamcinolone acetate intra- } \\
\text { articular (46) and extra- } \\
\text { articular (24) }\end{array}$ & $\begin{array}{l}\text { Method section: patients } \\
\text { were asked to report } \\
\text { appearance of flushing and } \\
\text { any abnormality of the } \\
\text { menstrual pattern Results: } \\
\text { shown in a table }\end{array}$ & $\begin{array}{l}\text { Disturbance in } \\
\text { menstruation at } 6 \text { weeks } \\
(50.6 \%) \text {, flushes }(28.6 \%)\end{array}$ & $\begin{array}{l}100 \% \\
\text { follow-up } \\
\text { after } \\
6 \text { weeks }\end{array}$ \\
\hline
\end{tabular}

RCT: randomized controlled trial, CT: controlled trial, Pros: prospective clinical study

- pain after injection with a frequency ranging from 3.4-81\%

- numbness and tingling in hands was reported in one study on CTS patients in $5 \%$ of the cases

- mild discoloration of the skin over the site of injection in three studies in $3.2 \%, 6 \%$ and $11.2 \%$, respectively

- disturbance in menstruation in one study in $50.6 \%$ of the patients, and flushes in 3 studies with a frequency of $3.2 \%, 22 \%$ and $28.6 \%$, respectively

-transient sympathetic reaction in one study with a frequency of $2 \%$

- ecchymosis in one study with a frequency of $1.3 \%$

Major adverse events in the prospective studies were:
- skin depigmentation reported in 3 studies with a frequency ranging from $1.3-4 \%$

- atrophy was mentioned in 5 studies with a frequency ranging from $1.5-40 \%$

- cellulitis was reported in one study in $4 \%$ of the patients

\section{Discussion}

In this review, reported dermal adverse events of local corticosteroid injections were irritation, change of skin colour, skin and perilymphatic atrophy, soft tissue calcification, skin defect, hypopigmentation, sterile abscess, ecchymosis, and allergic rash. The infectious adverse

Table 2 Summary of the included retrospective studies

\begin{tabular}{|c|c|c|c|c|}
\hline $\begin{array}{l}\text { Author, Year } \\
\text { of publication }\end{array}$ & Type of study & Indication & Corticosteroid used & Complication (number of cases) \\
\hline $\begin{array}{l}\text { Berthelot } 2004 \\
{[62]}\end{array}$ & $\begin{array}{l}\text { Questionnaire sent to } \\
500 \text { rheumatologist }\end{array}$ & $\begin{array}{l}\text { Different rheumatologic } \\
\text { diseases }\end{array}$ & $\begin{array}{l}\text { Cortivazol Hydrocortisone } \\
\text { Betamethasone } \\
\text { Paramethasone }\end{array}$ & Tachon's syndrome $(n=318) *$ \\
\hline Cill 2004 [98] & 48 cases & Achilles tendinopathy & $\begin{array}{l}\text { Triamcinolone } 10 \mathrm{mg} \text { and } 20 \\
\mathrm{mg} \text { with bupivacaine } 0.25 \%\end{array}$ & $\begin{array}{l}\text { No major complications, } 1 \text { patient (2\%) reported } \\
\text { purple skin dicoloration }\end{array}$ \\
\hline $\begin{array}{l}\text { Bjorkman } 2004 \\
\text { [99] }\end{array}$ & 27 cases & $\begin{array}{l}\text { Rupture of the tendon } \\
\text { extensor pollicis longus }\end{array}$ & $\begin{array}{l}2 \text { oral corticosteroids and } 2 \\
\text { local corticosteroid injections }\end{array}$ & $\begin{array}{l}\text { Rupture of the tendon extensor pollicis longus } \\
n=4 \text { associated with use of corticosteroids }\end{array}$ \\
\hline $\begin{array}{l}\text { Acevedo } 1998 \\
\text { [100] }\end{array}$ & 765 cases & Plantar fasciitis & Triamcinolone 40 mg (122) & Plantar fascia rupture $(n=44) * *$ \\
\hline $\begin{array}{l}\text { Astrom } 1998 \\
\text { [101] }\end{array}$ & 298 cases & Achilles tendinopathy & Unknown & $\begin{array}{l}\text { Preoperative steroid injection was predictive of } \\
\text { a partial rupture*** }\end{array}$ \\
\hline $\begin{array}{l}\text { Weinstein } 1984 \\
\text { [61] }\end{array}$ & Follow-up of 47 cases & $\begin{array}{l}\text { Traumatic olecranon } \\
\text { bursitis }\end{array}$ & $\begin{array}{l}25 \text { patients received } \\
\text { Triamcinolone } 20 \mathrm{mg} \text { after } \\
\text { aspiration }\end{array}$ & $\begin{array}{l}\text { septic bursitis (9\%) skin atrophy (25\%) chronic } \\
\text { pain }(28 \%)\end{array}$ \\
\hline
\end{tabular}

\footnotetext{
* 1 event per 8,000 injections

** 44 of the 51 plantar fascia ruptures were associated with corticosteroids injection

***Odds ratio 2.0 (Cl 1.3-9.8)
} 
events were cellulites, localized abscess, septic bursitis, atypical mycobacterium infection, necrotizing fasciitis, and protothecosis. Local adverse events included local pain, tingling or numbness in hands, local neural damage and tendon rupture. Systemic adverse events included allergic reactions, facial flush and disturbance in menstrual pattern.

Edwards and Aronson defined an adverse drug reaction as "an appreciably harmful or unpleasant reaction, resulting from an intervention related to the use of a medicinal product, which predicts hazard from future administration and warrants prevention or specific treatment, or alteration of the dosage regimen, or withdrawal of the product" [63]. According to the WHO they classify adverse drug reactions into six types: dose-related, non dose-related, dose-related and time-related, timerelated, withdrawal, and failure of therapy. In the present review we were unable to categorize the adverse drug reaction in this way. We neither were able to judge the causal relation between the reported reactions and the administered drug, so we are about speaking of adverse events rather than adverse drug reactions. In addition, in table 1 we reported the terminology as described in the individual articles, because it was not always clear which classification system they used. Although the adverse events reported in our review are 'miscellaneous' ones, we think that these types of adverse events (occurring after a regular dose of extraarticular corticosteroid injections) can be divided in systemic adverse events and local adverse events. The systemic adverse events can be divided into allergic reactions (IgE mediated) or other hypersensitivity reactions, disturbance in menstruation, flushes and Tachon's syndrome and systemic infection. The local effects consisted of local pain, degeneration, atrophy and change in skin colour, local infection, impact on collagen metabolism expressed as tendon ruptures, and perilymphatic atrophy. Depending on the place where the injection is administered, adverse events can manifest, for example, injections for plantar fasciitis are almost painful [64]. Injections in general can cause substantial adverse effects. For example, Nicolau's syndrome (livedoid dermatitis secondary to acute arterial thrombosis after injection in a blood vessel) has been described after an intra-articular corticosteroid injection [65]. Such an adverse event would be extremely rare after injection in a bursa or other superficial structures. The venous counterpart, known as Tachon's syndrome (subacute back pain and/or thoracic pain following local injections of corticosteroids), was reported in a retrospective study [62]. In general it obvious that adverse events associated with corticosteroid injection can be minimised by ensuring appropriate injecting procedures are followed by a well-trained practitioner. Neural damage after injecting
CTS might be avoided by proper injection technique [66]. In this review we divided the adverse events into minor ones (the harm was temporary) and major ones (the adverse event needed intervention or was not transient). This clinical categorization, although not approved by the WHO or FDA, might help to make a more balanced decision regarding the (possible) harm of an injection with corticosteroids for extra articular use. In addition, it can be easily explained to patients.

The Cochrane Collaboration provides guidance from the Adverse Effects Subgroup of the Non-randomized Studies Methods Group [13]. An appendix provides information on adverse effects, advice and tips about the search strategy and the type of studies to be included. However, we failed to find all the relevant articles with the search strategy advised by the Cochrane Collaboration and had to expand the search strategy. In our review, we did not use an overall quality assessment. We did describe however, the methods of reporting adverse events for each prospective study, the duration of follow-up, and the percentage lost to follow-up. The drawback of our study is that we could not assess the risk of bias. Clinical trials, cohort studies and case studies have their own risk of bias [13]. The limitations of the case reports are that there is uncertainty as to the adverse event was caused by the corticosteroid injection. Similarly, the lack of a control group in the prospective study on reporting specified menstruation disorders afterwards cannot prove the causal relationship [59]. If we assume that the internal validity for assessing adverse events in RCT at least should be based on the percentage available for follow-up (i.e. $80 \%$ or more) and systematic registration of adverse events and a comparison against a control group, then using these criteria less than half of the prospective studies in this review were of inferior quality.

Some RCTs assess smaller numbers of patients thus decreasing the chance of detecting a rare adverse event. Moreover, a part of the RCTs cover a relatively short study period thus precluding the identification of delayed or prolonged, and generally have highly specific inclusion/exclusion criteria that may imply that the results cannot be generalized to other populations.

Therefore, the assessment of safety needs to cover not only RCTs but also explore other sources such as, for example, post-marketing surveillance studies, spontaneous reporting schemes, and epidemiological studies. Systematic reviews on the safety of therapeutic interventions should preferably combine data from various types of studies [67].

In prospective studies, adverse effects attributed to the specific intervention should preferably be estimated by risk ratios, where the risk for adverse effects in the intervention group is compared with that for those who 
did not receive the intervention. In the present review, because all subjects included in the prospective studies received the intervention, only the percentage of adverse effects could be estimated. For this reason, in the RCTs we estimated the percentage of adverse effects for the intervention group only and did not compare these data with the control group. However, because the types of adverse event reported in these RCTs were highly intervention-specific we do not expect an overestimation of the adverse effects. In fact, based on the inadequate/lack of systematic registration in the included studies, we suspect there may even be an underestimation of the adverse-effects. Therefore we advocate that future RCTs and prospective studies should report on adverse events following the recommendations in the CONSORT guidelines [68].

\section{Conclusion}

In this literature review it was difficult to accurately quantify the incidence of adverse effects after extraarticular corticosteroid injection. Although one fatal adverse event after an extra-articular corticosteroid injection was reported, extra-articular corticosteroid injections are regularly administered worldwide. In the present review the incidence of major adverse events (according to our definition) was up to $5.8 \%$, ranging from depigmentation and atrophy of the skin to cellulitis; generally speaking these adverse effects could perhaps be classified as 'relatively mild'. Based on these data the administration of extra-articular corticosteroid injections seems to be a 'relatively safe' intervention.

\section{Additional material}

Additional file 1: Search strategy in Pubmed and Embase. Word DOC displaying search strategy in Pubmed and Embase.

\section{Acknowledgements}

This study is part of a thesis. The effect of corticosteroid injections are studied in a randomized trial. We tried to figure the risk of adverse effects of the injection therapy to consider the advantage of corticosteroid injection therapy.

We thank the Netherlands Pharmacovigilance Centre "Lareb" for providing us the reported adverse events.

All authors are employed at the Erasmus MC.

\section{Author details}

'Department of General Practice Erasmus Medical Center P.O. Box 2040, 3000 CA The Netherlands. ${ }^{2}$ Medical Library Erasmus Medical Center P.O. Box 2040, 3000 CA The Netherlands. ${ }^{3}$ Department of Orthopaedics, Erasmus Medical Center P.O. Box 2040, 3000 CA The Netherlands.

\section{Authors' contributions}

$A B$ designed the search strategy and read the abstracts and wrote the manuscript, AV carried out the search strategy in Pubmed and Embase, SB participated in its design and coordination and read a part of the abstracts and have been involved in drafting the manuscript. BK and JV participated in its design and coordination.

All authors have read and approved the final manuscript.

\section{Competing interests}

The authors declare that they have no competing interests.

Received: 1 September 2010 Accepted: 13 September 2010 Published: 13 September 2010

\section{References}

1. Wrenn RN, Goldner JL, Markee JL: An experimental study of the effect of cortisone on the healing process and tensile strength of tendons. J Bone Joint Surg Am 1954, 36-A(3):588-601.

2. Ines LP, da Silva JA: Soft tissue injections. Best Pract Res Clin Rheumatol 2005, 19(3):503-527.

3. Cardone DA, Tallia AF: Joint and soft tissue injection. American family physician 2002, 66(2):283-288

4. Cole BJ, Schumacher HR Jr: Injectable corticosteroids in modern practice. J Am Acad Orthop Surg 2005, 13(1):37-46.

5. GIPdatabank. [http://www.gipdatabank.nl/].

6. Lievense A, Bierma-Zeinstra S, Schouten B, Bohnen A, Verhaar J, Koes B: Prognosis of trochanteric pain in primary care. Br J Gen Pract 2005, 55(512):199-204.

7. Assendelft WJ, Hay EM, Adshead R, Bouter LM: Corticosteroid injections for lateral epicondylitis: a systematic overview. Br J Gen Pract 1996, 46(405):209-216

8. Spies-Dorgelo MN, van der Windt DA, Prins AP, Uitdehaag BM, van der Horst HE: Diagnosis and management of patients with hand and wrist problems in general practice. The European journal of general practice 2009, 15(2):84-94.

9. Ines LP, da Silva JA: Soft tissue injections. Best Pract Res Clin Rheumatol 2005, 19(3):503-527.

10. Peters-Veluthamaningal C, Winters JC, Groenier KH, Jong BM: Corticosteroid injections effective for trigger finger in adults in general practice: a double-blinded randomised placebo controlled trial. Ann Rheum Dis 2008, 67(9):1262-1266.

11. Nichols AW: Complications associated with the use of corticosteroids in the treatment of athletic injuries. Clin J Sport Med 2005, 15(5):370-375.

12. McIntosh HM, Woolacott NF, Bagnall AM: Assessing harmful effects in systematic reviews. BMC Med Res Methodol 2004, 4:19.

13. Higgins JPT, Green Se: Cochrane Handbook for Systematic Reviews of Interventions 5.0.2 [updated September 2009]. The Cochrane Collaboration 2008. 2008 [http://www.cochrane-handbook.org]

14. CBG MEB. [http://www.cbg-meb.nl/cbg/en/default.htm].

15. Derry S, Kong Loke $Y$, Aronson JK: Incomplete evidence: the inadequacy of databases in tracing published adverse drug reactions in clinical trials. BMC Med Res Methodol 2001, 1:7.

16. Schweitzer DH, Le-Brun PP, Krishnaswami S, Derendorf H: Clinical and pharmacological aspects of accidental triamcinolone acetonide overdosage: a case study. Neth J Med 2000, 56(1):12-16.

17. Hofmeister E, Engelhardt S: Necrotizing fasciitis as complication of injection into greater trochanteric bursa. Am J Orthop 2001, 30(5):426-427.

18. Yam A, Teoh LC, Yong FC: Necrotising fasciitis after corticosteroid injection for trigger finger: a severe complication from a 'safe' procedure. J Hand Surg Eur Vol 2009, 34(5):689-690.

19. Nanda V, Parwaz MA, Handa S: Linear hypopigmentation after triamcinolone injection: a rare complication of a common procedure. Aesthetic Plast Surg 2006, 30(1):118-119.

20. Evans AV, McGibbon DH: Symmetrical hypopigmentation following triamcinolone injection for de Quervain's tenosynovitis. Clin Exp Dermatol 2002, 27(3):247-251.

21. Friedman SJ, Butler DF, Pittelkow MR: Perilesional linear atrophy and hypopigmentation after intralesional corticosteroid therapy. Report of two cases and review of the literature. J Am Acad Dermatol 1988, 19(3):537-541.

22. Saour S, Dhillon BS, Ho-Asjoe M, Mohanna PN: Ascending hypopigmentation of the forearm following injection of triamcinolone. $J$ Plast Reconstr Aesthet Surg 2009, 62(12):e597-598. 
23. Lund IM, Donde R, Knudsen EA: Persistent local cutaneous atrophy following corticosteroid injection for tendinitis. Rheumatol Rehabil 1979, 18(2):91-93.

24. Okere $\mathrm{K}$, Jones MC: A case of skin hypopigmentation secondary to a corticosteroid injection. South Med J 2006, 99(12):1393-1394.

25. Kravette MA: Perilymphatic atrophy of skin. An adverse side effect of intralesional steroid injections. Clin Podiatr Med Surg 1986, 3(3):457-462.

26. Basadonna PT, Rucco V, Gasparini D, Onorato A: Plantar fat pad atrophy after corticosteroid injection for an interdigital neuroma: a case report. Am J Phys Med Rehabil 1999, 78(3):283-285.

27. Reddy PD, Zelicof SB, Ruotolo C, Holder J: Interdigital neuroma. Local cutaneous changes after corticosteroid injection. Clin Orthop Relat Res 1995, 317: 185-187

28. Civelek B, Celebioglu S: An unexpected complication of steroid use for the treatment of hypertrophic scar. Ann Plast Surg 2005, 54(2):221-222.

29. Jawed S, Allard SA: Osteomyelitis of the humerus following steroid injections for tennis elbow. Rheumatology (Oxford) 2000, 39(8):923-924.

30. Gidumal R, Evanski P: Calcaneal osteomyelitis following steroid injection: a case report. Foot Ankle 1985, 6(0):44-46.

31. Saglam N, Akpinar F: Intratendinous Septic Abscess of the Achilles Tendon after Local Steroid Injection. J Foot Ankle Surg 2009, 48(5):565-568.

32. Buccilli TA Jr, Hall HR, Solmen JD: Sterile abscess formation following a corticosteroid injection for the treatment of plantar fasciitis. J Foot Ankle Surg 2005, 44(6):466-468

33. Walsh SV, Johnson RA, Tahan SR: Protothecosis: an unusual cause of chronic subcutaneous and soft tissue infection. Am J Dermatopathol 1998, 20(4):379-382.

34. Baack BR, Brown RE: Atypical mycobacterium soft-tissue infection of the dorsal radial wrist: a possible complication of steroid injection for de Quervain's disease. Ann Plast Surg 1991, 27(1):73-76.

35. Miguelez A, Mestre F, Martin A, Escalas J, Del Pozo LJ: Allergic reaction to intralesional Celestone Cronodose. Br J Dermatol 2003, 149(4):894-896.

36. Gottlieb NL, Riskin WG: Complications of local corticosteroid injections. JAMA 1980, 243(15):1547-1548.

37. Smith AG, Kosygan $K$, Williams $H$, Newman RJ: Common extensor tendon rupture following corticosteroid injection for lateral tendinosis of the elbow. Br J Sports Med 1999, 33(6):423-424, discussion 424-425.

38. Fitzgerald BT, Hofmeister EP, Fan RA, Thompson MA: Delayed flexor digitorum superficialis and profundus ruptures in a trigger finger after a steroid injection: a case report. J Hand Surg Am 2005, 30(3):479-482.

39. Chen SK, Lu CC, Chou PH, Guo LY, Wu WL: Patellar tendon ruptures in weight lifters after local steroid injections. Arch Orthop Trauma Surg 2009, 129(3):369-372.

40. Chechick A, Amit $Y$, Israeli A, Horoszowski $H$ : Recurrent rupture of the achilles tendon induced by corticosteroid injection. Br J Sports Med 1982 , 16(2):89-90

41. Jones JG: Achilles tendon rupture following steroid injection. J Bone Joint Surg Am 1985, 67(1):170.

42. Linke E: [Achilles tendon ruptures following direct cortisone injection] Achillessehnenrupturen nach direkter Cortisoninjection. Hefte Unfallheilkd 1975, , 121: 302-303

43. Bedi SS, Ellis W: Spontaneous rupture of the calcaneal tendon in rheumatoid arthritis after local steroid injection. Ann Rheum Dis 1970, 29(5):494-495.

44. Ford LT, DeBender J: Tendon rupture after local steroid injection. South Med J 1979, 72(7):827-830.

45. Payne JM, Brault JS: Digital ischemia after carpal tunnel injection: a case report. Arch Phys Med Rehabil 2008, 89(8):1607-1610.

46. Swindells MG, Tehrani $H$, Goodwin-Walters A, Sassoon EM: Acute radial artery ischemia following therapeutic steroid injection. Ann Plast Surg 2007, 58(4):461-462.

47. Tavares SP, Giddins GE: Nerve injury following steroid injection for carpal tunnel syndrome. A report of two cases. J Hand Surg Br 1996, 21(2):208-209.

48. Kasten SJ, Louis DS: Carpal tunnel syndrome: a case of median nerve injection injury and a safe and effective method for injecting the carpal tunnel. J Fam Pract 1996, 43(1):79-82.

49. McConnell JR, Bush DC: Intraneural steroid injection as a complication in the management of carpal tunnel syndrome. A report of three cases. Clin Orthop Relat Res 1990, 250: 181-184.
50. Conti RJ, Shinder M: Soft tissue calcifications induced by local corticosteroid injection. J Foot Surg 1991, 30(1):34-37.

51. Friemann J, Mogilevski G, Hohr D, Rosorius H: [Calcifying granulomatous peritendinitis after local dexamethasone treatment] Kalzifizierende granulomatose Peritendinitis nach lokaler Dexamethasonbehandlung. Pathologe 1997, 18(6):459-462.

52. Raghavendran RR, Peart F, Grindulis KA: Subcutaneous calcification following injection of triamcinolone hexacetonide for plantar fasciitis. Rheumatology (UK) 2008, 47(12):1838.

53. Tonks JH, Pai SK, Murali SR: Steroid injection therapy is the best conservative treatment for lateral epicondylitis: a prospective randomised controlled trial. Int J Clin Pract 2007, 61(2):240-246.

54. Bisset $L$, Beller $E$, Jull $G$, Brooks P, Darnell R, Vicenzino B: Mobilisation with movement and exercise, corticosteroid injection, or wait and see for tennis elbow: randomised trial. Bmj 2006, 333(7575):939.

55. Jirarattanaphochai K, Saengnipanthkul S, Vipulakorn K, Jianmongkol S, Chatuparisute $P$, Jung S: Treatment of de Quervain disease with triamcinolone injection with or without nimesulide. A randomized, double-blind, placebo-controlled trial. J Bone Joint Surg Am 2004, 86A(12):2700-2706.

56. Tonks JH, Pai SK, Murali SR: Steroid injection therapy is the best conservative treatment for lateral epicondylitis: A prospective randomised controlled trial. Int J Clin Pract 2007, 61(2):240-246.

57. Hay EM, Paterson SM, Lewis M, Hosie G, Croft P: Pragmatic randomised controlled trial of local corticosteroid injection and naproxen for treatment of lateral epicondylitis of elbow in primary care. Bmj 1999, 319(7215):964-968

58. Price $\mathrm{R}$, Sinclair $\mathrm{H}$, Heinrich I, Gibson T: Local injection treatment of tennis elbow-hydrocortisone, triamcinolone and lignocaine compared. $\mathrm{Br} J$ Rheumatol 1991, 30(1):39-44.

59. Mens JM, Nico de Wolf A, Berkhout BJ, Stam HJ: Disturbance of the menstrual pattern after local injection with triamcinolone acetonide. Ann Rheum Dis 1998, 57(11):700.

60. Hui AC, Wong S, Leung CH, Tong P, Mok V, Poon D, Li-Tsang CW, Wong LK, Boet $R$ : A randomized controlled trial of surgery vs steroid injection for carpal tunnel syndrome. Neurology 2005, 64(12):2074-2078.

61. Weinstein PS, Canoso JJ, Wohlgethan JR: Long-term follow-up of corticosteroid injection for traumatic olecranon bursitis. Ann Rheum Dis 1984, 43(1):44-46.

62. Berthelot JM, Tortellier L, Guillot P, Prost A, Caumon JP, Glemarec J, Maugars $Y$ : Tachon's syndrome (suracute back and/or thoracic pain following local injections of corticosteroids). A report of 318 French cases. Joint Bone Spine 2005, 72(1):66-68.

63. Edwards $I R$, Aronson JK: Adverse drug reactions: definitions, diagnosis, and management. Lancet 2000, 356(9237):1255-1259.

64. Kalaci A, Cakici H, Hapa O, Yanat AN, Dogramaci Y, Sevinc TT: Treatment of plantar fasciitis using four different local injection modalities: a randomized prospective clinical trial. J Am Podiatr Med Assoc 2009, 99(2):108-113.

65. Cherasse A, Kahn MF, Mistrih R, Maillard H, Strauss J, Tavernier C: Nicolau's syndrome after local glucocorticoid injection. Joint Bone Spine 2003, 70(5):390-392.

66. Dubert T, Racasan O: A reliable technique for avoiding the median nerve during carpal tunnel injections. Joint Bone Spine 2006, 73(1):77-79.

67. Ernst $\mathrm{E}$, Pittler $\mathrm{MH}$ : Assessment of therapeutic safety in systematic reviews: literature review. Bmj 2001, 323(7312):546.

68. Altman DG, Schulz KF, Moher D, Egger M, Davidoff F, Elbourne D, Gotzsche PC, Lang T, Consort G: The revised CONSORT statement for reporting randomized trials: explanation and elaboration. Annals of internal medicine 2001, 134(8):663-694.

69. Rompe JD, Segal NA, Cacchio A, Furia JP, Morral A, Maffulli N: Home training, local corticosteroid injection, or radial shock wave therapy for greater trochanter pain syndrome. Am J Sports Med 2009, 37(10):1981-1990.

70. Gunter P, Schwellnus MP: Local corticosteroid injection in iliotibial band friction syndrome in runners: a randomised controlled trial. $\mathrm{Br} J$ Sports Med 2004, 38(3):269-272, discussion 272.

71. Chao M, Wu S, Yan T: The effect of miniscalpel-needle versus steroid injection for trigger thumb release. J Hand Surg Eur Vol 2009, 34(4):522-525. 
72. Peters-Veluthamaningal C, Winters JC, Groenier KH, Jong BM: Corticosteroid injections effective for trigger finger in adults in general practice: a double-blinded randomised placebo controlled trial. Ann Rheum Dis 2008, 67(9):1262-1266.

73. Jianmongkol S, Kosuwon W, Thammaroj T: Intra-tendon sheath injection for trigger finger: the randomized controlled trial. Hand Surg 2007, 12(2):79-82.

74. Goldfarb CA, Gelberman RH, McKeon K, Chia B, Boyer Ml: Extra-articular steroid injection: early patient response and the incidence of flare reaction. J Hand Surg [Am] 2007, 32(10):1513-1520.

75. Baumgarten KM, Gerlach D, Boyer MI: Corticosteroid injection in diabetic patients with trigger finger. A prospective, randomized, controlled double-blinded study. J Bone Joint Surg Am 2007, 89(12):2604-2611.

76. Kazuki K, Egi T, Okada M, Takaoka K: Clinical outcome of extrasynovial steroid injection for trigger finger. Hand Surg 2006, 11(1-2):1-4.

77. Gurcay E, Unlu E, Gurcay AG, Tuncay R, Cakci A: Evaluation of the effect of local corticosteroid injection and anti-inflammatory medication in carpal tunnel syndrome. Scott Med J 2009, 54(1):4-6.

78. Nalamachu S, Crockett RS, Mathur D: Lidocaine patch 5 for carpal tunnel syndrome: how it compares with injections: a pilot study. J Fam Pract 2006, 55(3):209-214.

79. Dammers JW, Roos Y, Veering MM, Vermeulen M: Injection with methylprednisolone in patients with the carpal tunnel syndrome: a randomised double blind trial testing three different doses. J Neurol 2006, 253(5):574-577.

80. Wong SM, Hui AC, Lo SK, Chiu JH, Poon WF, Wong L: Single vs. two steroid injections for carpal tunnel syndrome: a randomised clinical trial. Int J Clin Pract 2005, 59(12):1417-1421

81. Agarwal V, Singh R, Sachdev A, Wiclaff, Shekhar S, Goel D: A prospective study of the long-term efficacy of local methyl prednisolone acetate injection in the management of mild carpal tunnel syndrome. Rheumatology (Oxford) 2005, 44(5):647-650.

82. Ly-Pen D, Andreu JL, de Blas G, Sanchez-Olaso A, Millan I: Surgical decompression versus local steroid injection in carpal tunnel syndrome: a one-year, prospective, randomized, open, controlled clinical trial. Arthritis Rheum 2005, 52(2):612-619.

83. Sevim S, Dogu O, Camdeviren H, Kaleagasi H, Aral M, Arslan E, Milcan A: Long-term effectiveness of steroid injections and splinting in mild and moderate carpal tunnel syndrome. Neurol Sci 2004, 25(2):48-52.

84. Armstrong T, Devor W, Borschel L, Contreras R: Intracarpal steroid injection is safe and effective for short-term management of carpal tunnel syndrome. Muscle Nerve 2004, 29(1):82-88.

85. Wong SM, Hui AC, Tang A, Ho PC, Hung LK, Wong KS, Kay R, Li E: Local vs systemic corticosteroids in the treatment of carpal tunnel syndrome. Neurology 2001, 56(11):1565-1567.

86. Porter MD, Shadbolt B: Intralesional corticosteroid injection versus extracorporeal shock wave therapy for plantar fasciopathy. Clin J Sport Med 2005, 15(3):119-124.

87. Genc H, Saracoglu M, Nacir B, Erdem HR, Kacar M: Long-term ultrasonographic follow-up of plantar fasciitis patients treated with steroid injection. Joint Bone Spine 2005, 72(1):61-65.

88. Lindenhovius A, Henket M, Gilligan BP, Lozano-Calderon S, Jupiter JB, Ring D: Injection of Dexamethasone Versus Placebo for Lateral Elbow Pain: A Prospective, Double-Blind, Randomized Clinical Trial. J Hand Surg (USA) 2008, 33(6):909-919.

89. Wang AA, Whitaker E, Hutchinson DT, Coleman DA: Pain levels after injection of corticosteroid to hand and elbow. Am J Orthop 2003, 32(8):383-385

90. Smidt N, van der Windt DA, Assendelft WJ, Deville WL, Korthals-de Bos IB, Bouter LM: Corticosteroid injections, physiotherapy, or a wait-and-see policy for lateral epicondylitis: a randomised controlled trial. Lancet 2002, 359(9307):657-662.

91. Jensen B, Bliddal H, Danneskiold-Samsoe B: [Comparison of two different treatments of lateral humeral epicondylitis-"tennis elbow". A randomized controlled trial]. Ugeskr Laeger 2001, 163(10):1427-1431.

92. Stahl S, Kaufman T: The efficacy of an injection of steroids for medial epicondylitis: A prospective study of sixty elbows. J BONE JT SURG SER A 1997, 79(11):1648-1652.

93. Verhaar JA, Walenkamp GH, van Mameren $H$, Kester AD, van der Linden AJ: Local corticosteroid injection versus Cyriax-type physiotherapy for tennis elbow. The Journal of bone and joint surgery 1996, 78(1):128-132.
94. Avci S, Yilmaz C, Sayli U: Comparison of nonsurgical treatment measures for de Quervain's disease of pregnancy and lactation. J Hand Surg [Am] 2002, 27(2):322-324.

95. Anderson BC, Manthey R, Brouns MC: Treatment of De Quervain's tenosynovitis with corticosteroids. A prospective study of the response to local injection. Arthritis Rheum 1991, 34(7):793-798.

96. Crawford F, Atkins D, Young P, Edwards J: Steroid injection for heel pain: evidence of short-term effectiveness. A randomized controlled trial. Rheumatology (Oxford) 1999, 38(10):974-977.

97. Capasso G, Testa V, Maffulli N, Bifulco G: Aprotinin, corticosteroids and normosaline in the management of patellar tendinopathy in athletes: $A$ prospective randomized study. SPORTS EXERC INJ 1997, 3(3):111-115.

98. Gill SS, Gelbke MK, Mattson SL, Anderson MW, Hurwitz SR: Fluoroscopically guided low-volume peritendinous corticosteroid injection for Achilles tendinopathy. A safety study. J Bone Joint Surg Am 2004, 86-A(4):802-806.

99. Bjorkman A, Jorgsholm P: Rupture of the extensor pollicis longus tendon: a study of aetiological factors. Scand J Plast Reconstr Surg Hand Surg 2004, 38(1):32-35.

100. Acevedo Jl, Beskin JL: Complications of plantar fascia rupture associated with corticosteroid injection. Foot Ankle Int 1998, 19(2):91-97.

101. Astrom M: Partial rupture in chronic achilles tendinopathy: $A$ retrospective analysis of 342 cases. Acta Orthop Scand 1998, 69(4):404-407.

Pre-publication history

The pre-publication history for this paper can be accessed here: http://www.biomedcentral.com/1471-2474/11/206/prepub

\section{doi:10.1186/1471-2474-11-206}

Cite this article as: Brinks et al:: Adverse effects of extra-articular corticosteroid injections: a systematic review. BMC Musculoskeletal Disorders 2010 11:206.

\section{Submit your next manuscript to BioMed Central and take full advantage of:}

- Convenient online submission

- Thorough peer review

- No space constraints or color figure charges

- Immediate publication on acceptance

- Inclusion in PubMed, CAS, Scopus and Google Scholar

- Research which is freely available for redistribution

Submit your manuscript at www.biomedcentral.com/submit
C Biomed Central 